\title{
Single-port robotic radical cystectomy with ileal conduit urinary diversion: technique and review of the early outcomes in literature
}

\author{
Grace Chen, Simone Crivellaro \\ Department of Urology, University of Illinois at Chicago, Chicago, IL 60612, USA.
}

Correspondence to: Dr. Simone Crivellaro, Department of Urology, University of Illinois at Chicago, 1740 W Taylor Ave., Chicago, IL 60612, USA. E-mail: crivellaro76@hotmail.com

How to cite this article: Chen G, Crivellaro S. Single-port robotic radical cystectomy with ileal conduit urinary diversion: technique and review of the early outcomes in literature. Mini-invasive Surg 2021;5:54. https://dx.doi.org/10.20517/25741225.2021 .69

Received: 22 May 2021 First Decision: 23 Jun 2021 Revised: 26 Jul 2021 Accepted: 22 Oct 2021 Published: 17 Nov 2021

Academic Editor: Riccardo Autorino Copy Editor: Yue-Yue Zhang Production Editor: Yue-Yue Zhang

\begin{abstract}
The introduction of the da Vinci single port (SP) surgical system (Intuitive Surgical, Sunnyvale, CA, USA) has meant a necessary evolution in the surgical techniques used to perform various Urologic surgeries, such as roboticassisted radical cystectomy (RARC). In this paper, we describe a step-by-step technique for RARC with intracorporeal ileal conduit urinary diversion using the SP system at our institution and summarize early outcomes in the literature. The surgery was performed utilizing the standard institutional approach for radical cystectomy for the multiport robot, modified for the SP where appropriate. A total of 3 articles were found that included early patient outcomes after SP RARC. Including our institution, a total of 21 patients were included in the final analysis. The average patient age was 68 years old, 16 of the 21 patients were male, 13 of the patients had intracorporeal urinary diversions, the average operative time was $366 \mathrm{~min}$ with an average estimated blood loss of 185 . The average length of stay was 5.4 days. Among these patients, there were three 30-day complications noted and five 90-day complications, all of which were Clavian II or lower. We conclude that RARC utilizing the SP approach is both feasible and offers several theoretical advantages over the open and multiport approaches, but further study is necessary before advocating for widespread adoption of this modality.
\end{abstract}

Keywords: Single-port, robotic cystectomy, radical cystectomy, minimally invasive surgery 


\section{INTRODUCTION}

Muscle-invasive bladder cancer is an aggressive disease associated with high morbidity and mortality, and is primarily treated with radical cystectomy with multiple possible avenues for urinary diversion, including the ileal conduit urinary diversion or the orthotopic neobladder. This is classically done in an open fashion with the open radical cystectomy (ORC); however, with the increasing popularity of robotic surgery for pelvic surgery, there is growing interest in robotic-assisted radical cystectomy (RARC) as a minimally invasive alternative, with the use of the robot for radical cystectomy increasing from $16.7 \%$ in 2010 to $25.3 \%$ in 2013 in the United States ${ }^{[1]}$. The robots typically utilized for RARC include multiport generations of the da Vinci platform, including the da Vinci SI and XI systems.

The da Vinci single port (SP) robotic system (Intuitive Surgical, Sunnyvale, CA, USA) is the most recent robotic platform approved by the FDA in 2018 for urological surgery, and was designed with several modifications to the previously available multi-port robotic systems. The SP system combines the camera and all instrument arms into a single port, allowing surgery to be performed using a single incision. Other notable features include a relocation feature that allows the operator to reach all abdominal quadrants by moving the entire trocar with the attached arm around its fulcrum, and a virtual navigator that provides real-time monitoring of the relative position of the instruments, even when off the visual field. This allows for greater control of the instruments and safer positioning. Theoretical advantages of the SP platform over the multiport include improved cosmesis due to surgery being performed through a single incision. Fewer incisions also have a theoretical benefit of reduced pain and improved visualization in a narrow space such as the pelvis. Retrospective studies on robotic prostatectomy have already shown an advantage in terms of pain scores after surgery and length of stay, with comparable outcomes ${ }^{[2,3]}$.

This article illustrates the technique performed utilizing the SP robotic system for the robotic-assisted laparoscopic radical cystectomy with ileal conduit urinary diversion in a male patient. To date, there are only three other published papers detailing the use of the SP robot for RARC, and no noninferiority studies comparing the SP RARC to multiport RARC. We provide a step-by-step technical approach to surgery with special attention paid to technical modifications from the multi-port technique.

\section{METHODS}

We provide a review of our technique for single-port radical cystectomy based on the experience from our institution. A video of the procedure is available as well. A review of early outcomes has been carried out through a retrospective analysis of clinical documentation. A systematic review of the literature outcomes was performed via a broad search of PubMed using the following keywords: da Vinci SP, single port robotic cystectomy, and radical cystectomy. We included a single patient from our institution, who was chosen according to standard patient selection for RARC, including the ability to tolerate pneumoperitoneum and steep Trendelenburg, BMI $<30 \mathrm{~kg} / \mathrm{m}^{2}$, and lack of prior pelvic radiation or trauma ${ }^{[4]}$.

\section{Step-by-step surgical technique}

Patient positioning and port placement

After induction of general anesthesia, the patient is positioned in the standard positioning for robotic pelvic surgery, specifically the dorsal lithotomy position with arms tucked, extremities padded and secured, and the bed in steep Trendelenburg. After the patient is prepped and draped in the normal sterile fashion, a Foley catheter is placed. A $2.5 \mathrm{~cm}$ incision is then made inferolateral to the umbilicus, approximately $1 / 3$ of the distance between the umbilicus and iliac crest, and the Hasson technique is used to dissect through layers of fascia to access the abdominal cavity. Of note, this incision is later used as our stoma site for ileal conduit creation. We then insert an Alexis retractor into this incision site and attach the GelPOINT 
advanced access platform (Applied Medical, Rancho Santa Margarita, CA, USA) with the SP Cannula to the Alexis. The abdomen is then insufflated to $15 \mathrm{mmHg}$, and carefully surveyed to identify any abdominal adhesions. We then place 2 additional trocars under direct vision, specifically a $12 \mathrm{~mm}$ port (to which an AirSeal is attached) approximately $2 / 3$ of the distance between the umbilicus and left iliac crest and a $5 \mathrm{~mm}$ assistant port halfway between the umbilicus and the 12 port. The SP robot is then side-docked.

\section{General considerations}

The SP RARC technique largely follows the standard multiport technique with RARC, with several key adjustments, notably the positioning of the robotic instruments. We begin with the monopolar scissors at the 3 o'clock position, Cardiere forceps at the 6 o'clock position, bipolar forceps at the 9 o'clock position, and the camera at the 12 o'clock position. Instruments are switched periodically to allow for optimal retraction depending on the specific step of the procedure performed.

\section{Identification of the ureters}

We locate the ureters bilaterally by incising the overlying peritoneum just lateral to the medial umbilical ligament and dissecting down to the level of the common iliac artery. The Cadiere forceps at 6 o'clock are useful for holding traction on the ureters and pushing the bowel medially during this dissection, which can be completed without the bedside assistant. Once the ureters are identified, they are placed on vessel loops and dissected down to the ureteropelvic junction, at which time they are clipped with two Hem-o-lock clips (Weck Closure Systems, Research Triangle Park, NC, USA) and divided.

\section{Anterior and posterior bladder dissection}

The LigaSure device is then used to divide the obliterated umbilical arteries bilaterally and the tissue lateral to the ureters. The bipolar forceps at 9 o'clock are used to lift the bladder, the Cardiere at 6 o'clock is used to provide downward traction on the bladder, and the monopolar scissors at 3 o'clock are used to open up the endopelvic fascia. The posterior peritoneum is then incised over the rectum, connecting the two entry points into the endopelvic fascia. The seminal vesicles are then identified and elevated, and the posterior plane between the rectum and bladder is bluntly dissected out. The LigaSure device is used to divide the superior vesical arteries bilaterally, taking care to stay below the seminal vesicles and ureteral stumps bilaterally. This dissection plane is taken all the way down to the prostate apex. We then divide the median and medial umbilical ligaments using the LigaSure device and drop the bladder into the pelvis. After the apex of the prostate is dissected, we switch our 3 o'clock and 9 o'clock instruments out for robotic needle drivers and oversew Santorini's plexus using 2-0 V-Loc suture in a figure-of-eight fashion. We then replace our monopolar scissors at 3 o'clock and bipolar forceps at 9 o'clock and divide Santorini's plexus. Next, we dissect out and free the urethra. The Foley catheter is clipped with a Weck clip to keep the balloon inflated to avoid spillage of bladder contents. The remaining lateral attachments of the prostate are then divided in a modified nerve-sparing fashion with bipolar cautery. Once the specimen is completely freed, it is placed in a $15 \mathrm{~mm}$ entrapment sac and set aside.

\section{Pelvic lymphadenectomy}

We then perform our bilateral pelvic lymphadenectomy, removing the external, obturator, internal, and common iliac lymph nodes bilaterally using Weck clips and bipolar cautery for lymphostasis. We periodically switch the positions of the Cadiere and bipolar forceps between the 6 o'clock and 9 o'clock positions as needed for better retraction of the lymph nodes, as the Cardiere provides a better medial retraction. The specimens are sent to pathology as right and left pelvic lymph nodes. The presacral space is then divided to allow for the passage of the left ureter under the mesorectum at the level of the sacral promontory. This dissection is performed with the Cardiere forceps at the 9 o'clock position to hold 
traction on the sigmoid colon.

\section{Intracorporeal ileal conduit creation}

We begin the intracorporeal reconstruction portion of the case with the bipolar forceps at the 6 o'clock position, needle driver at 9 o'clock, and Cadiere forceps at 3 o'clock. The left ureter is tunneled under the presacral space and sigmoid colon, with the Cadiere forceps pulling the ureter through into the right retroperitoneum. The ileocecal valve is then identified, and a 3-0 Vicryl stay stitch is placed $30 \mathrm{~cm}$ from the valve, with a $15 \mathrm{~cm}$ segment of ileum marked out for the conduit. At this time, we switch the needle driver to the 3 o'clock position and Cadiere to 9 o'clock, and monopolar scissors at 6 o'clock. Ligasure is used to take down the mesentery. The monopolar scissors are used to open up the bowel at both ends. The EndoGIA stapler is advanced through the 12 trocar assistant port, and a stapled side-to-side small bowel anastomosis is performed at the first and second corners of each end of the bowel. This is first stapled across longitudinally, and then stapled again to seal the edge of the side-to-side anastomosis. Additional 3-0 Vicryl is used to buttress the staple line. We then orient the proximal segment of the ileal conduit towards the pelvis and the distal end towards the skin.

The right ureter is trimmed, with the distal ureter sent for frozen pathology. The ureter is then spatulated and anastomosed to the conduit in an end-to-side fashion with a running 4-0 Vicryl in a Bricker style. This is done with the Cadiere at the 6 o'clock position to hold the conduit down and the bipolar forceps holding the ureter up - it must be noted that no assistant is needed for the anastomosis. We similarly prepare, spatulate, and anastomose the left ureter to the proximal end of the ileal conduit in an end-to-side fashion in the Bricker style.

Before each anastomosis is closed, we place single-J ureteral stents inside each ureter in the following fashion. Through the gel point, we insert a laparoscopic right angle holding a Motion wire inside the $2.5 \mathrm{~cm}$ right lower quadrant incision beside the robotic instruments. The wire is then passed from the distal to the proximal end of the conduit using the laparoscopic right angle, and pulled through with the Cadiere forceps. The wire is then advanced into the ureter, and the single-J stent is advanced over the wire up the ureter until resistance is felt. The same step is used to place a stent up the ureter. After the conclusion of the ureteral-ileal anastomoses, the single $\mathrm{j}$ is secured to the distal part of the conduit with a long 0 Vicryl suture, the tail of which can be followed through the gel point. Eventually, a 15 round JP drain is inserted into the pelvis via the $5 \mathrm{~mm}$ port, which we suture to the skin with a 2-0 nylon. The robot is then undocked, the pneumo removed, and the specimen is removed through the SP incision. If needed, the incision is lengthened slightly to accommodate the sample. At this point, pulling gently on the o Vicryl previously placed, we can recover the distal part of the conduit, grab it with a ring forceps and bring it out through the SP incision. The stoma is then secured with 3-0 Vicryl to the fascia with seromuscular bites, and then the end of the stoma is matured, securing it to the dermis with seromuscular to mucosa to dermal sutures circumferentially, taking care not to suture the mesentery of the ileum. The stents are then trimmed and brought into a urostomy bag.

\section{RESULTS}

A total of 3 articles were found in the literature that summarized early patient outcomes after SP RARC ${ }^{[5-7]}$. Including our institution, a total of 21 patients were included in the final analysis. The average patient age was 68 years old, 16 of the 21 patients were male, and 13 of the 21 patients had intracorporeal urinary diversions. The average operative time was $366 \mathrm{~min}$ with average estimated blood loss of 185 . Average length of stay was 5.4 days. Among these patients, there were three 30-day 188 complications noted and five 90-day complications, all of which were Clavian II or lower [Table 1]. 
Table 1. Early clinical outcomes

\begin{tabular}{|c|c|c|c|c|c|c|c|c|c|}
\hline Age & Sex & $\begin{array}{l}\text { Op } \\
\text { time }\end{array}$ & EBL & $\begin{array}{l}\text { Nodal } \\
\text { harvest }\end{array}$ & $\begin{array}{l}\text { Length } \\
\text { of stay }\end{array}$ & Preop path & Postop path & $\begin{array}{l}30 \text { day } \\
\text { complication }\end{array}$ & $\begin{array}{l}90 \text { day } \\
\text { complication }\end{array}$ \\
\hline 65 & Male & 411 & 250 & 4 & 7 & pTa high volume & pT1NO & Clavian I & None \\
\hline 89 & Male & 245 & 200 & 10 & 6 & & pT1 & None & \\
\hline 68 & Male & 285 & 250 & 16 & 5 & & pT2a & None & \\
\hline 67 & Male & 309 & 400 & 6 & 5 & & рT3b & Clavian II & \\
\hline 86 & Male & 242 & 150 & 18 & 6 & & pT2a & None & \\
\hline 70 & Female & 496 & 100 & 9 & 5 & $\begin{array}{l}\text { PT2 high grade, } \\
\text { micropapillary } \\
\text { features }\end{array}$ & pT4aNO UC & Clavian I (n/v) & \\
\hline 75 & Male & 475 & 300 & 12 & 5 & pT2 high grade UC & $\begin{array}{l}\text { pT2bNO, marg neg, pT2 } \\
\text { adenocarcinoma of prostate, } \\
\text { ISUP grade } 2\end{array}$ & None & \\
\hline 71 & Female & 420 & 100 & 18 & 5 & $\begin{array}{l}\text { pT1 high grade BCG } \\
\text { refractory }\end{array}$ & pTisNO UC, marg neg & None & \\
\hline 71 & Male & 425 & 750 & 8 & 5 & $\begin{array}{l}\text { pT1 high-grade, } \\
\text { inside bladder } \\
\text { diverticulum }\end{array}$ & $\begin{array}{l}\text { pT1NO, UC inside divertic, } \\
\text { margins neg }\end{array}$ & None & \\
\hline 64 & $\begin{array}{l}3 \text { females, } \\
9 \text { males }\end{array}$ & 387 & 117 & 11.9 & 5.4 & & $2 \mathrm{~T} 1 ; 10 \mathrm{~T} 2$ & & 5 \\
\hline
\end{tabular}

\section{DISCUSSION}

Urologists have always been early to adopt new technological advances in the field of surgery, and Urology was one of the first subspecialties to widely adopt the use of the da Vinci robot for various procedures involving the prostate, kidney, and bladder. Most notably, the use of the da Vinci robot has become so widespread for radical prostatectomy that it is now used for up to $85 \%$ of all radical prostatectomies ${ }^{[8]}$. Urologists have been comparatively slower to adopt the robot for use in radical cystectomies, owing at least in part to the cystectomy being a more complex and technically challenging procedure, particularly due to the need for bladder reconstruction and urinary diversion. In addition, operative times tend to be longer for the robotic cystectomy without current proven benefit in terms of local recurrence rates ${ }^{[9]}$. There is relatively little data currently out there on outcomes after SP RARC, owing in part to the newness of the SP system. Nevertheless, the addition of the da Vinci SP platform represents an exciting advancement in the realm of minimally invasive surgery, and with the rise in popularity and proven noninferiority of RARC compared to ORC, it is worth exploring and reporting the feasibility, safety, and outcomes of RARC utilizing the SP robot. The initial case reviews included in the study represent a promising start in demonstrating the safety and feasibility of performing RARC using the da Vinci SP robot.

\section{Conclusion}

RARC with intracorporeal ileal conduit urinary diversion can be performed in a safe manner with good preliminary outcomes using the new da Vinci SP platform. More studies with larger case volumes are required to determine distinguishing variables such as average length of procedure, length of hospital stay, complications, surgical margins, and post-operative local recurrence rates. Given numerous theoretical benefits of the SP system over multiport, including improved cosmesis, reduced pain requirements, and improved operative visualization in narrow spaces, it is an avenue of great interest in the field of minimally invasive Urology and warrants further exploration.

\section{DECLARATIONS}

\section{Authors' contributions}

Conceived the topic of study, provided guidance and expertise surrounding the technical steps of the procedure, and critically revised the final manuscript: Crivellaro $S$ 
Data gathering and literature review, data analysis and interpretation, and manuscript writing: Chen G

\section{Availability of data and materials}

Aside from the single patient from our institution included in the final data analysis of outcomes after SP RARC, all other data is officially published data accessible to any interested party. The data for outcomes outside of our institution is available through a basic PubMed search for SP RARC. Institution-specific data is available upon reasonable request.

\section{Financial support and sponsorship}

None.

\section{Conflicts of interest}

Dr. Simone Crivellaro is a consultant for Intuitive Surgical, Inc.

\section{Ethical approval and consent to participate}

Informed consent to participate in this study and similar studies was obtained from all patients undergoing robotic surgery at the University of Illinois at Chicago.

\section{Consent for publication}

Written consent for publication was obtained when the patient consented to participate in the study.

\section{Copyright}

(c) The Author(s) 2021.

\section{REFERENCES}

1. Pignot G, Treacy P, Walz J. Growing evidence for benefits of minimally invasive radical cystectomy. Transl Androl Urol 2020;9:245961. DOI PubMed PMC

2. Vigneswaran HT, Schwarzman LS, Francavilla S, Abern MR, Crivellaro S. A comparison of perioperative outcomes between singleport and multiport robot-assisted laparoscopic prostatectomy. Eur Urol 2020;77:671-4. DOI PubMed

3. Saidian A, Fang AM, Hakim O, Magi-Galluzzi C, Nix JW, Rais-Bahrami S. Perioperative outcomes of single vs multi-port robotic assisted radical prostatectomy: a single institutional experience. J Urol 2020;204:490-5. DOI PubMed

4. Balbay MD, Koc E, Canda AE. Robot-assisted radical cystectomy: patient selection and special considerations. Robot Surg 2017;4:1016. DOI PubMed PMC

5. Kaouk J, Garisto J, Eltemamy M, Bertolo R. Single-port robotic intracorporeal ileal conduit urinary diversion during radical cystectomy using the sp surgical system: step-by-step technique. Urology 2019;130:196-200. DOI PubMed

6. Zhang M, Thomas D, Salama G, Ahmed M. Single port robotic radical cystectomy with intracorporeal urinary diversion: a case series and review. Transl Androl Urol 2020;9:925-30. DOI PubMed PMC

7. Gross JT, Vetter JM, Sands KG, et al. Initial experience with single-port robot-assisted radical cystectomy: comparison of perioperative outcomes between single-port and conventional multiport approaches. J Endourol 2021;35:1177-83. DOI PubMed

8. İnkaya A, Tahra A, Sobay R, Kumcu A, Küçük EV, Boylu U. Comparison of surgical, oncological, and functional outcomes of robotassisted and laparoscopic radical prostatectomy in patients with prostate cancer. Turk J Urol 2019;45:410-7. DOI PubMed PMC

9. Sathianathen NJ, Kalapara A, Frydenberg M, et al. Robotic assisted radical cystectomy vs open radical cystectomy: systematic review and meta-analysis. J Urol 2019;201:715-20. DOI PubMed 